
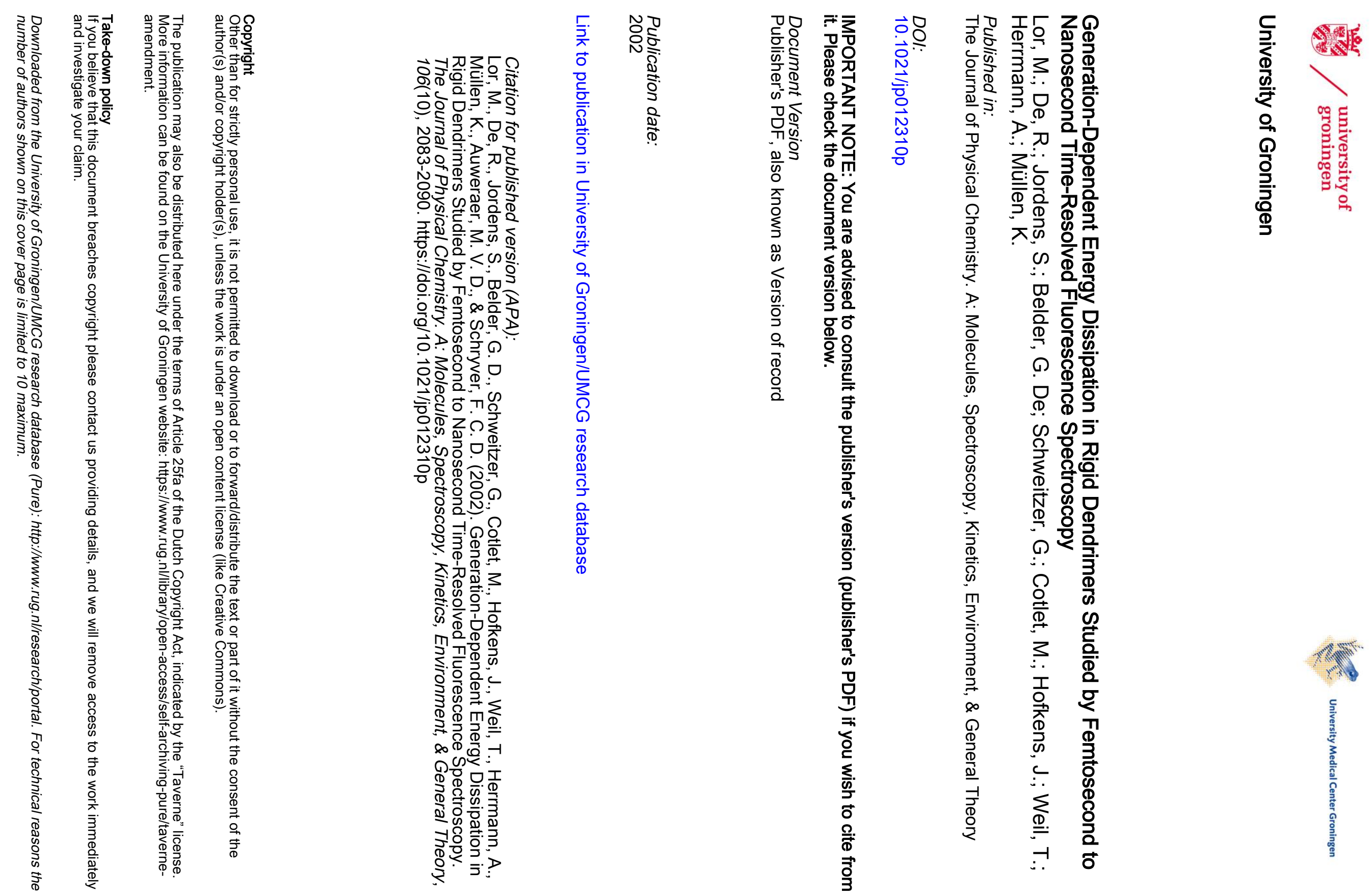


\title{
Generation-Dependent Energy Dissipation in Rigid Dendrimers Studied by Femtosecond to Nanosecond Time-Resolved Fluorescence Spectroscopy ${ }^{\dagger}$
}

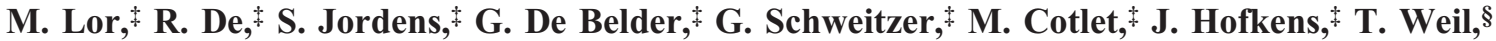 \\ A. Herrmann, $§$ K. Miillen, $₫$ M. Van Der Auweraer, ${ }^{\ddagger}$ and F. C. De Schryver* $*$ \\ Department of Chemistry, Katholieke Universiteit Leuven, Celestijnenlaan 200 F, 3001 Heverlee, Belgium, and \\ Max-Planck-Institut für Polymerforschung, Ackermannweg 10, 55128 Mainz, Germany
}

Received: June 19, 2001; In Final Form: August 29, 2001

\begin{abstract}
Intramolecular kinetic processes in a series of second-generation polyphenyl dendrimers with multiple peryleneimide chromophores attached to the para position of the outer phenyl ring were investigated by steadystate and femtosecond to nanosecond time-resolved fluorescence spectroscopy. The results obtained were compared to the ones of the corresponding first-generation dendrimer series. The energy-hopping rate constant, $k_{\text {hopp }}$, observed from anisotropy decay times was found to be 5 times smaller than that of the first-generation series and scales well with the difference in average distance between the chromophores. In addition to the processes observed in first-generation dendrimers in the ultrafast time domain by fluorescence up-conversion, a second annihilation process is found in the second-generation multichromophoric dendrimer. The observation of two singlet-singlet annihilation processes in this compound can be explained by the presence of a mixture of constitutional isomers leading to a broader distribution of distances between neighboring chromophores compared to first-generation multichromophoric dendrimers.
\end{abstract}

\section{Introduction}

Dendrimers are highly branched three-dimensional macromolecular systems, which in contrast to other macromolecules can be defined on a molecular level ${ }^{1,2}$ in size, shape, and functionality. For this reason, dendrimers have been attracting much attention not only from the synthetic point of view ${ }^{3}$ but also from the point of view of their physical and chemical properties. $^{4-9}$ Dendrimers consist of an interior building block (core) surrounded by successive branched layers, the so-called generations, and a periphery. ${ }^{10} \mathrm{~A}$ broad range of applications for dendrimers has been reported in several fields such as guesthost chemistry, ${ }^{11}$ analytical chemistry, ${ }^{12}$ optoelectronics, ${ }^{13}$ catalysis, ${ }^{14}$ biology, ${ }^{15}$ and medicine. ${ }^{16}$ It has also been shown that dendrimers can mimic natural light-harvesting antenna systems. ${ }^{17-19}$

Within the research group, two series of first-generation dendrimers with an identical rigid central $\mathrm{sp}^{3}$ core and substituted with peryleneimide chromophores at the meta and para position of the outer phenyl ring have been investigated at the single-molecule level ${ }^{20}$ as well as at the ensemble level. ${ }^{21-24}$ The studies mentioned above lead to the understanding of the time-resolved behavior of these dendrimers. In the time-resolved single-photon-counting measurements, the meta-substituted dendrimers showed the presence of some excited dimer-like states resulting from chromophore-chromophore interaction, whereas for the para-substituted ones, no such state has been observed. Excitation of the peryleneimide chromophore results in excitation energy hopping among similar chromophores for both dendrimer series. The hopping rate constants were evaluated.

\footnotetext{
$\dagger$ Part of the special issue "Noboru Mataga Festschrift".

* To whom correspondence should be addressed. E-mail: Frans.DeSchryver@Chem.KULeuven.ac.be.

$\$$ Katholieke Universiteit Leuven.

$\S$ Max-Planck-Institut für Polymerforschung.
}

The short time scale dynamics has been studied by means of femtosecond fluorescence up-conversion. These measurements revealed four different kinetic processes for both first-generation dendrimers: an internal vibrational relaxation, a vibrational and solvent relaxation, an intermolecular interaction, and the fluorescence. A fifth process, singlet-singlet annihilation, is only present in the multichromophoric compounds as was established by an excitation energy-dependent study. ${ }^{21}$ It has been shown that the singlet-singlet annihilation process contributes to a larger extent in the para-substituted dendrimers than in the meta-substituted ones. ${ }^{21}$ These differences between the meta- and para-substituted dendrimers demonstrate the important role of the spatial distribution of the chromophores at the periphery in the dynamics of the photophysical processes involved.

To determine the influence of the generation and size of these dendrimers on the annihilation process and energy hopping, a series of second-generation para-substituted peryleneimide dendrimers $(\mathbf{G} 2 \mathbf{R} \boldsymbol{x} ; \boldsymbol{x}=1-4)$ with a rigid tetrahedral core was investigated (Figure 1A). In this study, we report the comparison between the solution-phase ensemble photophysics of a first and second generation of these dendrimers investigated by steadystate and nanosecond to femtosecond time-resolved spectroscopic techniques.

\section{Experimental Section}

Synthesis of G2Rx. G2R $\boldsymbol{x}$ was synthesized by way of repetitive Diels-Alder cycloadditions. The crucial intermediate in the synthesis is the functionalized tetraphenylmethane core ((4-diethynylphenyl)di-[4-(trisopropylsilyl)ethynylphenyl]methane core for G2R2, Figure 1C). ${ }^{25}$ With the dye (Figure 1B) attached to the para position of one phenyl group of structure 1A, the chromophoric group can be described as being connected to the innermost $\mathrm{sp}^{3}$ carbon center of the dendrimer

\subsection{1/jp012310p CCC: $\$ 22.00 \quad$ C 2002 American Chemical Society} Published on Web 10/10/2001 
A)

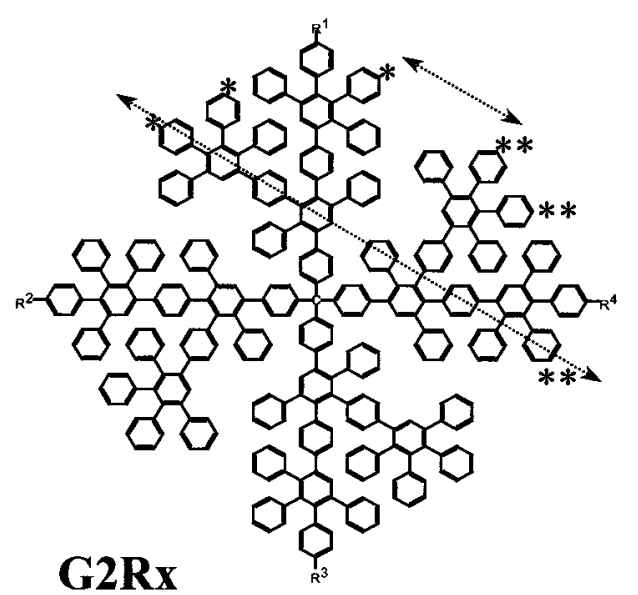

\begin{tabular}{lllll} 
& $\mathbf{R}^{\mathbf{1}}$ & $\mathbf{R}^{2}$ & $\mathbf{R}^{3}$ & $\mathbf{R}^{4}$ \\
\hline G2R1 & PI & H & H & H \\
G2R2 & PI & PI & H & H \\
G2R3 & PI & PI & PI & H \\
G2R4 & PI & PI & PI & PI
\end{tabular}

B)

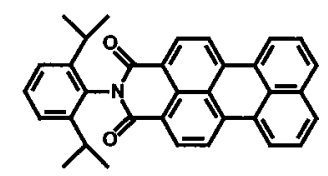

PI

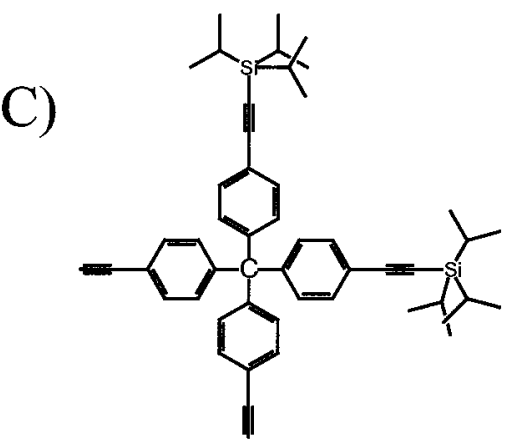

Figure 1. Molecular structures of the molecules used in this study: (A) G2Rx ( $x=1,2,3,4)$, second-generation dendrimers; (B) PI, peryleneimide chromophore; (C) difunctionalized tetraphenylmethane core. Different positions for chromopohore attachment are marked with $*$ and $* *$.

via a rigid pentaphenylene chain. It must be pointed out though that as a result of the Diels-Alder cycloaddition each dye can, in principle, adopt four different positions on a dendrimer branch, thus leading to sixteen constitutional isomers for G2R2. Inspection of the steric situation (Figure 1A and Figure 2a', $\mathrm{b}^{\prime}$ ) reveals clearly, however, that these different spatial locations lead to different classes of bischromophores where the two extremes (short and long distance) have an average minimum (maximum) distance of ca. 28 (37) A. These distances have been obtained by calculating the interchromophoric distance of each constitutional isomer and determining the two average extreme values based on three-dimensional structures obtained by Merck molecular force field calculations. ${ }^{26}$

All samples were dissolved in toluene (Aldrich). The integrity of all samples was checked thoroughly by taking steady-state absorption and emission spectra before and after each set of measurements. No photodegradation was observed.

Steady-State Measurements. Steady-state absorption and corrected fluorescence spectra were recorded with Lambda 40
(Perkin-Elmer) and Fluorolog (SPEX) spectrophotometers, respectively. The solutions obtained by dissolving the dendrimers in toluene (Aldrich) had an optical density below 0.1 at the absorption maximum $(495 \mathrm{~nm})$ in a $1 \mathrm{~cm}$ cuvette, which corresponds to a concentration of about $10^{-7} \mathrm{M}$. The fluorescence quantum yields have been determined using a peryleneimide-substituted polyphenylene dendrimer (meta-G1R1) as a reference. ${ }^{23}$

Picosecond Time-resolved Experiments. The fluorescence decay times of these second-generation dendrimers (G2R1, G2R2, G2R3, G2R4) have been determined by the singlephoton-counting method (SPC) using a setup described previously. ${ }^{27}$ In brief, the second harmonic of a Ti:sapphire laser (Tsunami, Spectra Physics) pumping an optical parametric oscillator (OPO) has been used to excite the samples at 530 $\mathrm{nm}$ with a repetition rate of $4 \mathrm{MHz}$. The detection system consists of a subtractive double monochromator (9030DS, Sciencetech) and a microchannel plate photomultiplier (R3809U, Hamamatsu). A time-correlated single-photon-counting PC module (SPC 630, Picoquant) was used to obtain the fluorescence decay histograms in 4096 channels with time increments of 5 or 10 ps. The fluorescence decays have been recorded at three different orientations of the emission polarizer relative to the polarization plane of the excitation light, i.e., $54.7^{\circ}, 0^{\circ}\left(I_{\mathrm{par}}\right)$, and $90^{\circ}\left(I_{\text {perp }}\right)$. The magic angle decays were analyzed globally with a time-resolved fluorescence analysis (TRFA) software. ${ }^{28}$ The quality of the fits has been judged by the fitting parameters such as $\chi^{2}(<1.2), Z \chi^{2}(<3)$, and the Durbin-Watson parameter $(1.8<\mathrm{DW}<2.2)$ as well as by the visual inspection of the residuals and autocorrelation function. ${ }^{29}$ The anisotropy decay analysis was performed with our global fluorescence decay analysis program TRFA, ${ }^{28}$ which takes pulse deconvolution into account.

Femtosecond Fluorescence Up-Conversion Experiments. These experiments were performed on the second-generation dendrimers containing one (G2R1) and four (G2R4) peryleneimide chromophores. All measurements on these two compounds were performed at room temperature in $1 \mathrm{~mm}$ optical path length cuvettes under magic angle polarization conditions (magic angle to eliminate complexity otherwise induced by anisotropy components). All compounds were dissolved in toluene at a concentration that yielded an absorption of ca. 0.4 per $\mathrm{mm}$ at the excitation wavelength of $495 \mathrm{~nm}$, corresponding to a concentration on the order of $10^{-5} \mathrm{M}$.

Besides the measurements under these standard conditions, one more series was performed in which the excitation energy was varied deliberately (see below).

The laser system has previously been described in detail. ${ }^{30}$ In brief, a Nd: $\mathrm{YVO}_{4}$ laser (Millennia V, Spectra Physics) is used to pump a Ti:sapphire laser (Tsunami, Spectra Physics). Its output seeds a regenerative amplifier (RGA, Spitfire, Spectra Physics). The output of the RGA ( $1 \mathrm{~mJ}, 100 \mathrm{fs}, 800 \mathrm{~nm}$ ) is split in two equal parts, one of which is used to pump an optical parametric generator/amplifier (OPA-800, Spectra Physics) and a second part that is used as a gate pulse. The output wavelength range of the OPA is extended by harmonic generation using one or two $\beta$-barium triborate (BBO) crystals, thus making a range of 300-900 $\mathrm{nm}$ accessible.

The fluorescence up-conversion detection setup has also been described in detail in a previous publication. ${ }^{31}$ Briefly, the fluorescence light emitted from the sample is collected and sent to a lithium borate (LBO) crystal, in which the sum frequency of this light and a gate pulse $(800 \mathrm{~nm}$, ca. $100 \mu \mathrm{J})$ derived from the RGA is generated. The time-resolved traces are then 

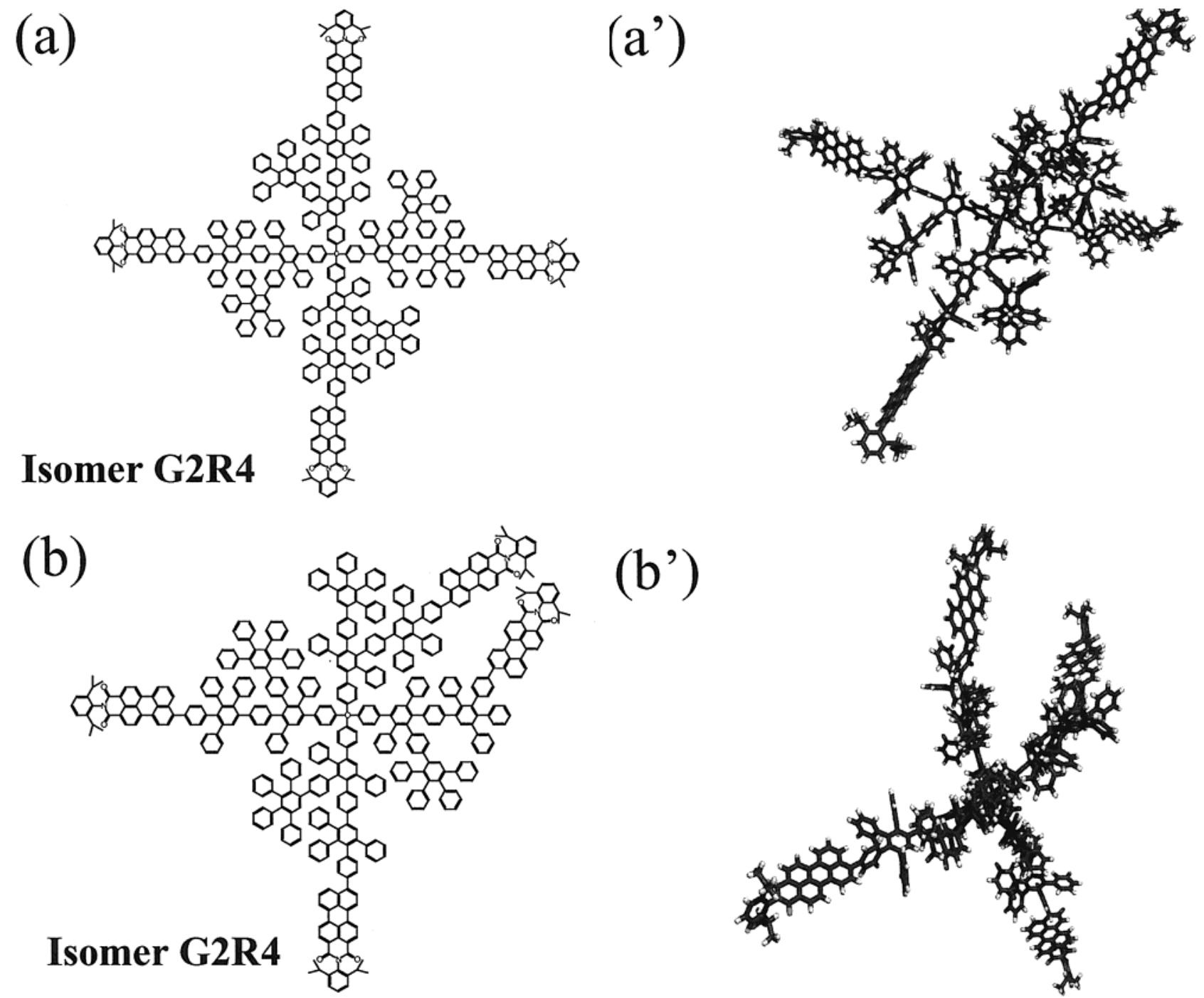

Figure 2. Molecular structures of G2R4 isomers: (a) isomer with a long-distance pair of chromophores; (a') 3D structure of isomer with a long-distance pair of chromophores; (b) isomer with a short-distance pair of chromophores; ( $b^{\prime}$ ) 3D structure of isomer with a short-distance pair of chromophores.

collected by detecting this sum frequency light while changing the relative delay of the gate pulse versus the sample excitation time. By detection of scattered light under otherwise identical conditions, the prompt response of this arrangement (including laser sources) was determined to be approximately $250 \mathrm{fs}$. This value was used in the analysis of all measurements for deconvolution of the data sets.

For all measurements performed here, the excitation wavelength was kept constant at $495 \mathrm{~nm}$. Except for the series checking the excitation energy dependence (see below), all compounds were excited with a pulse energy of ca. $400 \mathrm{~nJ}$. Each measurement consisted of 1024 delay positions, at each of which the fluorescence signal, the excitation laser intensity, and the gate pulse intensity were recorded averaging over five seconds, thus resulting in a measurement time of ca. $5000 \mathrm{~s}$ per delay scan.

To capture all kinetic components potentially present in the excited-state dynamics as precisely as possible, a measurement as described above was then repeated using three different channel widths for the detection. This results in three different time windows of $6.7,50$, and 450 ps for all 1024 measured channels. This set of three measurements was performed for each compound throughout its complete emission spectrum at 15 different fluorescence detection wavelengths from 530 to
$670 \mathrm{~nm}$ at intervals of $10 \mathrm{~nm}$, thus resulting in a set of 45 measurements.

To investigate possible multiphoton processes, an additional series of measurements was performed varying the laser energy exciting the compound in a systematic way, while keeping all other conditions constant. This was done in a range from 420 $\mathrm{nJ}$ (maximum laser output available) down to $20 \mathrm{~nJ}$, which turned out to be at the detection limit.

The data analysis was performed independently for each molecule, but in an identical fashion. All 45 decay curves measured in all three time windows were put together into a common data set, which was analyzed globally using a nonlinear least-squares fit routine from a commercial software package. As part of the analysis, the data sets were also deconvoluted using information from system prompt response measurements that were routinely recorded during each measurement session.

For all dendrimers, a sum of four exponentials with time constants $\tau_{1}-\tau_{4}$ and amplitudes $a_{1}-a_{4}$ has been found to be necessary to fit the data sets properly as judged by minimization of $\chi^{2}$ values and visual inspection of residual plots. The longest lived component $\left(\tau_{4}, a_{4}\right)$ was determined by a single-photoncounting detection setup and kept fixed in the analysis of the femtosecond up-conversion study. It has a time constant of 3.9 ns. 


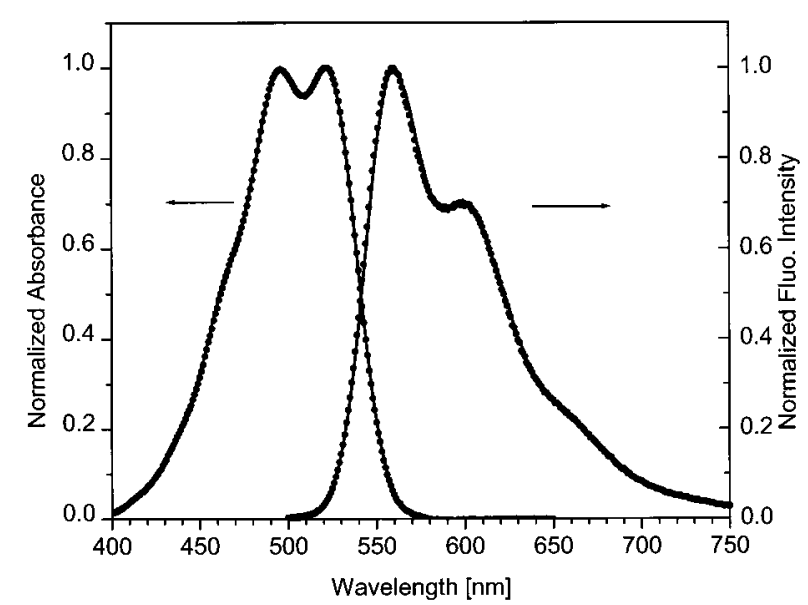

Figure 3. Normalized steady-state absorption and emission spectra for the model compound G2R1 (solid line) and G2R4 (dots). The emission spectra were excited at a wavelength of $495 \mathrm{~nm}$.

TABLE 1. Fluorescence Decay Times $\left(\tau^{a}\right)$ and Fit Parameters of the Fluorescence Anisotropy Decays Measured for the Rigid Dendrimers in Toluene with $\lambda_{\text {exc }}=$ $530 \mathrm{~nm}$ and $\lambda_{\mathrm{flu}}=600 \mathrm{~nm}$ and Average Peryleneimide-Peryleneimide Distances $\left(d_{\text {FRET }}\right)^{b}$

\begin{tabular}{ccccccccc}
\hline compound & $\tau^{a}(\mathrm{~ns})$ & $r_{0}$ & $\Theta_{1}$ & $\Theta_{2}(\mathrm{~ns})$ & $\beta_{1}$ & $\beta_{2}$ & $\beta_{2} / r_{0}(\%)$ & $d_{\text {FRET }}(\AA)$ \\
\hline G2R1 & 3.9 & 0.32 & 2.7 & & 0.32 & & & \\
G2R2 & 3.9 & 0.36 & 3.1 & 0.41 & 0.18 & 0.18 & 0.50 & 36 \\
G2R3 & 3.9 & 0.36 & 2.7 & 0.31 & 0.14 & 0.22 & 0.61 & 37 \\
G2R4 & 3.9 & 0.35 & 3.0 & 0.28 & 0.08 & 0.27 & 0.77 & 38
\end{tabular}

${ }^{a}$ Globally analyzed over different wavelengths between 550 and 700 nm. ${ }^{b} d_{\text {FRET }}$ is derived from eq 6 on the basis of the rate of energy hopping within the Förster energy-transfer model.

\section{Results}

Steady-State Spectra. The steady-state absorption and emission spectra of the rigid dendrimers (G2R1, G2R2, G2R3, G2R4) under investigation were found to be independent of the number of chromophores present in the dendrimers. Representative examples for the model compound with one chromophore, G2R1, and with a maximum number of four chromophores, G2R4, in toluene are shown in Figure 3. The absorption spectra show two vibronic maxima at 495 and 520 $\mathrm{nm}$. The fluorescence spectra give two vibronic maxima at 560 and $600 \mathrm{~nm}$. No spectral changes have been observed when compared with the corresponding first-generation dendrimer series. The fluorescence quantum yield $\left(\Phi_{\mathrm{f}}\right)$ is calculated to be $0.98 \pm 0.05$ and is identical within experimental error for all compounds.

Time-Resolved Fluorescence Measurements. To investigate the excited-state properties of these dendrimers, fluorescence decay times were measured in toluene by single-photon counting detecting the emission under the magic angle condition. The fluorescence decays were monitored at different emission wavelengths and were globally analyzed. All decays could be fitted globally to a single exponential with a decay time of 3.9 ns (Table 1).

Time-Resolved Fluorescence Polarization Measurements. From fluorescence anisotropy measurements, anisotropy relaxation times and the associated anisotropy values have been determined for G2R1, G2R2, G2R3, and G2R4 using the following well-known expression:

$$
r(t)=\sum \beta_{i} \exp \left(-t / \Theta_{i}\right) \quad \text { and } \quad r_{0}=\sum_{i} \beta_{i}
$$

TABLE 2. Decay Times Resulting from Global Analysis for All Compounds Investigated by Femtosecond Fluorescence Up-Conversion

\begin{tabular}{ccccc}
\hline compound & $\tau_{1}(\mathrm{ps})$ & $\tau_{2}(\mathrm{ps})$ & $\tau_{3}(\mathrm{ps})$ & $\tau_{4}{ }^{a}(\mathrm{ps})$ \\
\hline G1R1 & $0.5-2^{b}$ & 6.3 & 110 & 3900 \\
G1R4 & $0.5-2^{b}$ & 4.0 & 45 & 3900 \\
G2R1 & $0.5-2^{b}$ & 6.0 & 50 & 3900 \\
G2R4 & $0.5-2^{b}$ & 5.8 & 40 & 3900
\end{tabular}

${ }^{a}$ Fixed during the fit procedure. ${ }^{b}$ Varying with fluorescence detection wavelength.

For the dendrimers with more than one chromophore, a twoexponential function was found to be essential to fit the experimental anisotropy decay traces (Table 1). The long depolarization time constant relates to overall rotation of the molecule, whereas the short depolarization time constant, which decreases with the number of chromophores in the dendrimers, relates to energy hopping (vide infra).

Femtosecond Fluorescence Up-Conversion Measurements. To reveal ultrafast processes, femtosecond fluorescence upconversion measurements were performed. In a first series, the second-generation dendrimer containing one peryleneimide chromophore (G2R1) was investigated, serving as a model compound for a molecule with only one chromophore attached to a dendritic core. In a second series, the second-generation dendrimer containing four peryleneimide chromophores at the rim (G2R4) was studied. To investigate the influence of the generation number in detail, the results obtained with these second-generation dendrimers will be compared to those of the first-generation dendrimers containing one (G1R1) and four (G1R4) peryleneimide chromophores. ${ }^{21}$

By global analysis, four decay components were revealed in both second-generation compounds. Their decay times are summarized in Table 2.

As the decay time $\tau_{1}$ was not found to be constant throughout the spectrum, it could not be analyzed globally. In all cases, it varies between $500 \mathrm{fs}$ at short fluorescence detection wavelengths and 2 ps at longer fluorescence detection wavelengths. The behavior of this component is identical to the ultrafast decay component of the first-generation dendrimers (G1R1 and G1R4). This kinetic component was attributed to a process occurring within one chromophore, ${ }^{22}$ which is therefore independent of the generation number. Also, the ultrafast decay component recovered in the mono- and multichromophoric second-generation dendrimers under investigation can be attributed to this intramolecular vibrational-energy redistribution (IVR) process in the electronically excited state of the chromophore. ${ }^{32,33}$

The second component $\left(\tau_{2}, a_{2}\right)$ exhibits a fast time constant on the order of 6 ps for both second-generation compounds, G2R1 and G2R4. The time constant of the third component ranges from $40 \mathrm{ps}$ for $\mathbf{G 2 R} 1$ to $50 \mathrm{ps}$ for G2R4. The fourth decay time of 3.9 ns was determined by SPC measurements.

The amplitudes of the components determined by femtosecond fluorescence up-conversion are depicted in Figure 4A and 4B for G2R1 and G2R4, respectively. The amplitude of the second component represents $10 \%-40 \%$ of the total amplitude for both second-generation compounds, depending on the detection wavelength. The contribution of the third component to the total amplitude is grossly different in both secondgeneration compounds. For the multichromophoric G2R4, this third component is contributing between $20 \%$ and $35 \%$ to the total amplitude, depending on the detection wavelength. For the monochromophoric G2R1, this component is contributing at the most $10 \%$ of the total amplitude, again depending on the 


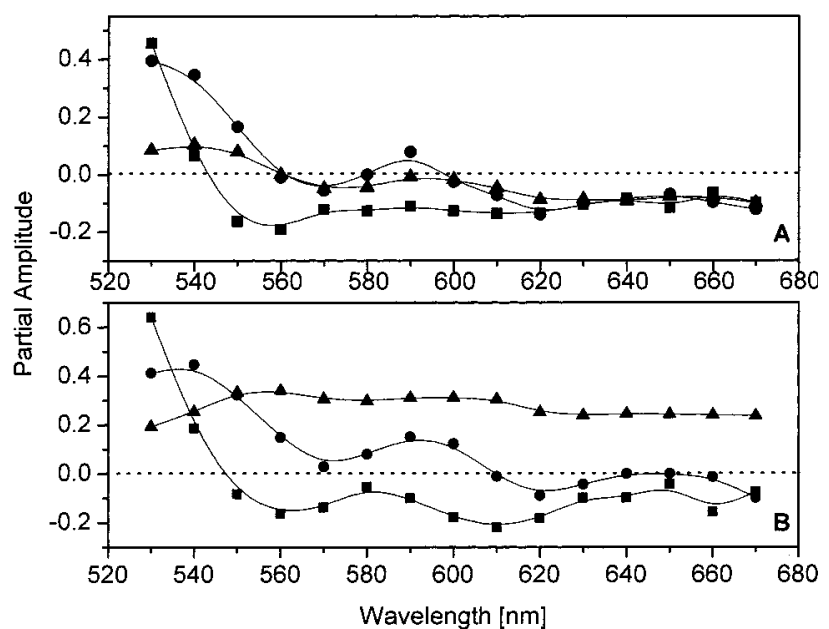

Figure 4. Wavelength dependence of the partial amplitudes $\left(a_{1}, a_{2}\right.$, $\left.a_{3}\right)$ of the decay times $\left(\tau_{1}, \tau_{2}, \tau_{3}\right)$ of the second-generation dendrimers determined by femtosecond fluorescence up-conversion: (A) monochromophoric second-generation compound G2R1; (B) multichromophoric second-generation compound G2R4; $\tau_{1}(\mathbf{\square}), \tau_{2}(\bullet), \tau_{3}(\mathbf{\Delta})$.

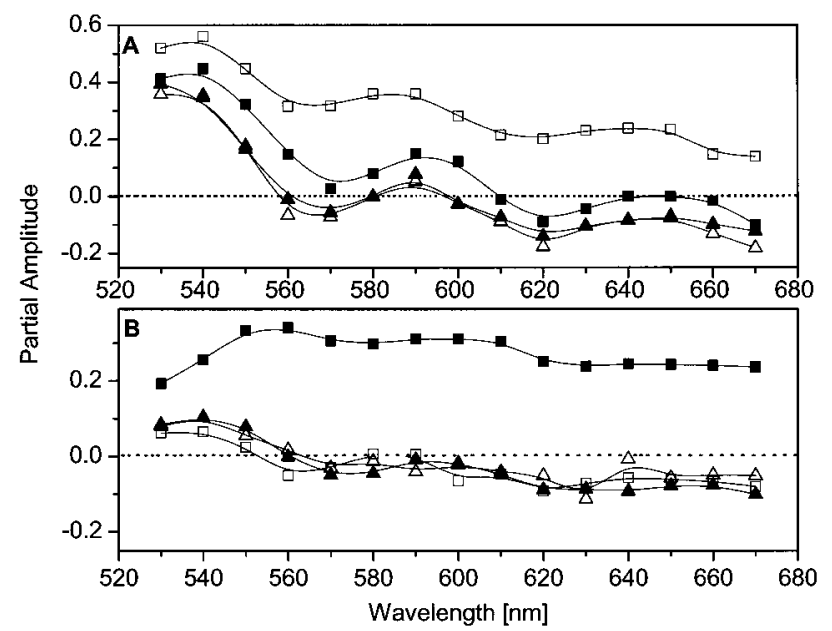

Figure 5. Wavelength dependence of the amplitude $a_{2}$ of the second component (A) and the amplitude $a_{3}$ of the third component (B) for

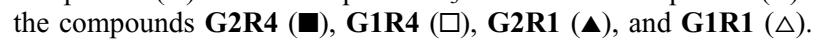

detection wavelength. The largest part of the amplitude, however, is found in the nanosecond component $\left(\tau_{4}, a_{4}\right)$ for both compounds (data not shown).

The second decay component that could be found back in the second-generation dendrimers has a decay time of $6 \mathrm{ps}$ (Table 2). Figure 5A shows the partial amplitudes for the monochromophoric second-generation compound G2R1 in function of the detection wavelength. From the shape and the positive/negative amplitude behavior, this kinetic component can be attributed to a vibrational and solvent relaxation in the electronically excited state of the peryleneimide chromophore. $22,34-40$

To investigate the influence of chromophore-chromophore interactions, the multichromophoric second-generation compound G2R4 was studied. The positive offset of the partial amplitude curves of G2R4 compared to those of G2R1 indicates that more than one process is contributing to the second decay component of the multichromophoric compound as opposed to the monochromophoric compound. ${ }^{21,22}$ The first pathway contributing to this process in both compounds has been attributed to a relaxation process (see above). The second pathway contributing to this process is an intramolecular singlet-singlet
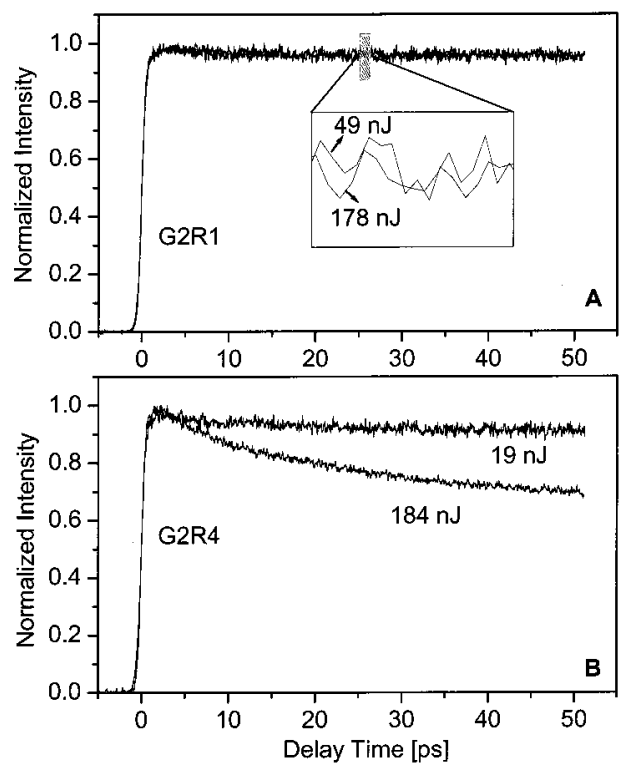

Figure 6. Comparison of the time-resolved fluorescence intensity recorded at low- and high-excitation energy (as indicated): (A) monochromophoric compound G2R1 detected at $590 \mathrm{~nm}$; (B) multichromophoric compound G2R4 detected at $590 \mathrm{~nm}$.

annihilation process that is independent of detection wavelength and exists only in compounds with multiple chromophores. ${ }^{41-46}$

To extend the hypothesis formulated for G1R4 and G2R4 and to be able to separate these two processes, an excitation energy-dependent study was performed on both second-generation compounds. By varying the excitation energy impinging on the sample between $20 \mathrm{~nJ}$ and $420 \mathrm{~nJ}$, a clear dependence of the amplitude of the $6 \mathrm{ps}$ component could be observed. This energy-dependent study was performed at the strategically chosen detection wavelength of $590 \mathrm{~nm}$. For the monochromophoric compound (G2R1), the partial amplitude for the second component is close to zero. However, for the multichromophoric compound (G2R4), the amplitude of the second component is shifted to a positive value. Thus, all intensity dependence observed at the $590 \mathrm{~nm}$ detection wavelength can be attributed to the intramolecular singlet-singlet annihilation process. The partial amplitudes for the multichromophoric compound (G2R4) are shifted to a higher value over the entire detection wavelength range. In Figure 6B, the decays recorded at the $590 \mathrm{~nm}$ detection wavelength and at different excitation energies are depicted for the multichromophoric G2R4. Because the relative importance of the annihilation process should increase as the excitation energy increases, the partial amplitudes as a function of the detection wavelength of the 6 ps component of the multichromophoric compound should at low excitation energy resemble the one for the monochromophoric compound. This is exactly what is observed (Figure 6B). The shift of the partial amplitude, $a_{2}$, over the whole wavelength range of the multichromophoric G2R4 is indicative of an annihilation process. ${ }^{22,42}$

A similar energy-dependent study has been performed for the monochromophoric G2R1. In Figure 6A, the results are depicted, and they show no detectable excitation energy dependence. The dependence of the partial amplitude $a_{2}$ of this $6 \mathrm{ps}$ component on the incident laser energy is shown in Figure 7A and 7B for G2R1 and G2R4 at 630 and $590 \mathrm{~nm}$ detection wavelengths, respectively. This is a clear indication that in G2R1 the annihilation process is absent and that the amplitude spectrum is only showing the vibrational and solvent relaxation of the chromophore itself. 
The same energy-dependence study has been performed on the third decay component. Figure 8A and 8B depicts the partial amplitude $a_{3}$ for G2R1 and G2R4 at 630 and $590 \mathrm{~nm}$, respectively, as a function of the excitation energy. The monochromophoric G2R1 shows no dependence on the excitation energy at the two selected detection wavelengths. This is in contrast to the results for the multichromophoric G2R4, which shows a clear dependence at these wavelengths (Figures $8 \mathrm{~A}$ and $8 \mathrm{~B}$ ). In view of the typical power dependence, the $40 \mathrm{ps}$ component of G2R4 can also be attributed to an annihilation process.

\section{Discussion}

In a previous study, ${ }^{21,24}$ a similar series of measurements was performed on the first-generation para-substituted polyphenylene dendrimers similarly substituted with the peryleneimide chromophore. By comparing these results with the newly obtained ones of the second-generation dendrimers, the influence of the generation number upon the kinetics can be revealed.

The similarity of the steady-state properties of all the dendrimers in terms of spectral shape and fluorescence maxima suggests that the emission occurs from the same state in all of the dendrimers. Table 1 shows that the decay traces for all of these can be fitted well by a monoexponential function. No long decay component of 7.4 ns as obtained with the meta-substituted first-generation rigid core dendrimer $(\boldsymbol{m}-\mathbf{G 1 R 1})^{23}$ was observed. Contrary to the meta compound, there are no isomers formed in which the two chromophores can approach each other sufficiently to form an excimer-like state.

Intramolecular Energy Hopping. The time-resolved anisotropy data can reveal the characteristic features of the energyhopping process. The multichromophoric dendrimers present two-exponential decays in the anisotropy traces. The fast component (410-280 ps) of the anisotropy decay (Table 1), which is found to decrease from $\mathbf{G} \mathbf{2} \mathbf{2}$ to $\mathbf{G} \mathbf{2} \mathbf{R} 4$, can be related to the excitonic energy hopping among identical chromophores. Considering the time scale, this process of energy hopping could be explained in the framework of the Förster theory as has been discussed in the meta and para series of dendrimers ${ }^{23,24}$ of the first generation. The effective interaction radius $\left(R_{0}\right)$ can be calculated from the steady-state spectra and the fluorescence quantum yield of the donor chromophore $\left(\phi_{\mathrm{D}}\right)$ with eqs. 2 and 3

$$
R_{0}^{6}=8.875 \times 10^{-5} \frac{\kappa^{2} \phi_{\mathrm{D}} J}{n^{4}}
$$

where $\kappa^{2}$, as a first approximation, is set to $2 / 3$ for the usually assumed random orientation of the chromophores, $\phi_{\mathrm{D}}$ is the donor fluorescence quantum yield, $n$ is the refractive index of the solvent (1.496 for toluene) and $J$ is the spectral overlap integral defined by

$$
J=\frac{\int F_{\mathrm{D}}(\lambda) \epsilon_{\mathrm{A}}(\lambda) \lambda^{4} \mathrm{~d} \lambda}{\int F_{\mathrm{D}}(\lambda) \mathrm{d} \lambda}
$$

where $\epsilon_{\mathrm{A}}(\lambda)$ represents the molar extinction coefficient of the acceptor and $\mathrm{F}_{\mathrm{D}}(\lambda)$ denotes the donor fluorescence spectrum on a wavelength $(\lambda)$ scale. Using the spectral data $\left(\epsilon_{\max } / 3=\right.$ $\left.38.300 \mathrm{M}^{-1} \mathrm{~cm}^{-125}, \phi_{\mathrm{f}}=98 \%\right)$, the calculated value of $J$ is found to be $2.5 \times 10^{14} \mathrm{M}^{-1} \mathrm{~cm}^{-1} \mathrm{~nm}{ }^{4}$

From eq 2 , it can be seen that the Förster radius $\left(R_{0}\right)$ strongly depends on the orientation factor $\kappa$ between the two interacting chromophores. As the attachment of the chromophores to the dendrimer backbone cannot be taken as random, the value of $\kappa$ has been calculated from the three-dimensional molecular structure,

$$
\kappa=\sin \left(\delta_{\mathrm{D}}\right) \sin \left(\delta_{\mathrm{A}}\right) \cos \left(\varphi_{\mathrm{DA}}\right)-2 \cos \left(\delta_{\mathrm{D}}\right) \cos \left(\delta_{\mathrm{A}}\right)
$$

where $\varphi_{\mathrm{DA}}$ is the azimuthal angle between the involved transition dipole moment directions of the energy donor $\mathrm{D}$ and acceptor $\mathrm{A}$ and $\delta_{\mathrm{D}}$ and $\delta_{\mathrm{A}}$ are the angles between the corresponding dipole directions of $\mathrm{D}$ and $\mathrm{A}$ with the internuclear $\mathrm{D}-\mathrm{A}$ axis, respectively. The average value of around 2.7 has been found for the dendrimers in which the chromophores are at long distance from each other (Figure 2a, $\mathrm{a}^{\prime}$ ). However, for the isomer of G2R4 with a short distance pair of chromophores (Figure $2 \mathrm{~b}, \mathrm{~b}^{\prime}$ ), the average $\kappa^{2}$ for all couplings between pairs of two chromophores is obtained as 1.5. Hence, the hopping rate constant, $k_{\text {hopp}}$, obtained from experimental results should be considered as an average hopping for the different possible constitutional isomers in the dendrimer.

Taking into account the possibility of multiple hopping channels in the multichromophoric systems containing identical chromophores, an average hopping rate constant ( $k_{\text {hopp }}$ ) according to the energy-hopping model ${ }^{23}$ is given by the expression

$$
k_{\text {hopp }}=\frac{1}{i x \Theta_{2}}-\frac{1}{i x \Theta_{1}}
$$

where $\Theta_{1}$ and $\Theta_{2}$ are the experimental extracted decay times and the value of $i$ represents the number of chromophores fully interacting in both the forward and the backward direction. Through the use of eq 5, a value of $0.85 \mathrm{~ns}^{-1}$ for $k_{\text {hopp }}$ of these dendrimers is obtained. This value is more than five times smaller than that of the corresponding first-generation dendrimers. The anisotropy contribution $\beta_{2} / r_{0}$ (Table 1 ) due to energy hopping can be verified, ${ }^{23}$ and the respective values of $\beta_{2} / r_{0}$ for G2R3 (61\%) and G2R4 (77\%) agree quite well with the theoretical values of $66 \%$ and $75 \%$, respectively, suggesting full cross-talk among all chromophores. The constancy, within experimental error, of the $r_{0}$, the sum of the two $\beta$ values, in this series is in contrast with the G1R $\boldsymbol{x}$ meta ${ }^{23}$ series of dendrimers but identical to the G1R $\boldsymbol{x}$ para $^{24}$ series and relates to the absence of structural conformers in which overlapping chromophores lead to excimer-like emission.

With the use of the excited-state lifetime $\left(\tau_{\mathrm{D}}\right)$, the derived values of $R_{0}(45 \AA)$ with a value of $\kappa^{2}$ of 2.7 and $k_{\text {hopp}}$, the distance between the two chromophores has been calculated from the following equation:

$$
d_{\mathrm{FRET}}{ }^{6}=\frac{R_{0}{ }^{6}}{k_{\mathrm{hopp}} \tau_{\mathrm{D}}}
$$

This yields a value of $d_{\text {FRET }}=37 \AA$, which is in good agreement with the average interchromophoric distance obtained from molecular modeling. From the sixth-power dependence of $k_{\text {hopp }}$ on the average interchromophoric distance $\left(d_{\text {FRET }}\right)$ and the use of the ratio of the values of $k_{\text {hopp }}$ for the first- and secondgeneration series, an average for $d_{\text {FRET }}$ for the second-generation series is found to be $37 \AA$. The decrease in hopping rate constant in these molecules hence scales with the sixth power of the distance difference as expected within the Förster model.

Intramolecular Singlet-Singlet Annihilation. In Figure 5A, the comparison between the amplitude spectra of $\tau_{2}$ of the multichromophoric and the monochromophoric dendrimers is shown as a function of the detection wavelength. The positive 


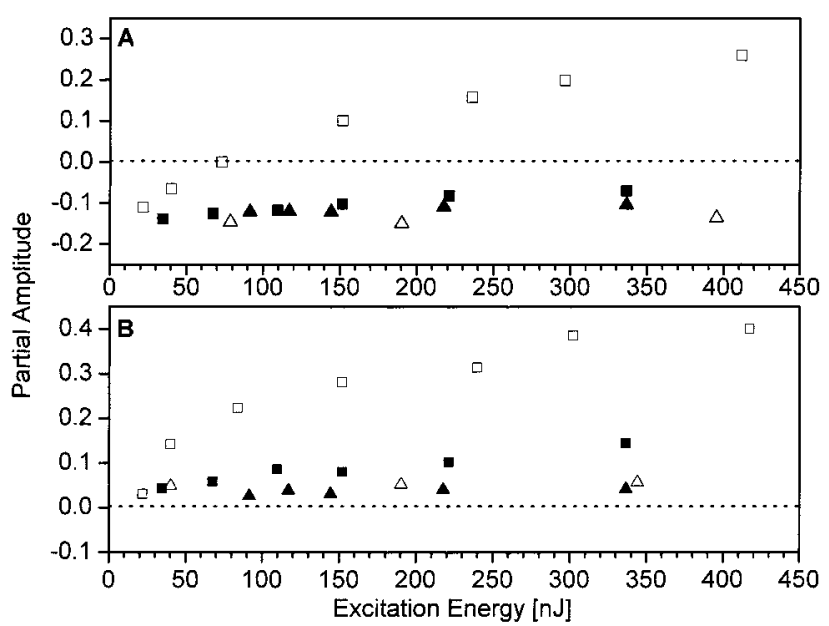

Figure 7. Dependence of the partial amplitude $a_{2}$ of the second component from the laser excitation energy for the compounds G2R4 $(\square)$, G2R1 ( $\triangle)$, G1R4 ( $\square)$, and G1R1 $(\triangle)$ at detection wavelength $630 \mathrm{~nm}(\mathrm{~A})$ and $590 \mathrm{~nm}(\mathrm{~B})$.

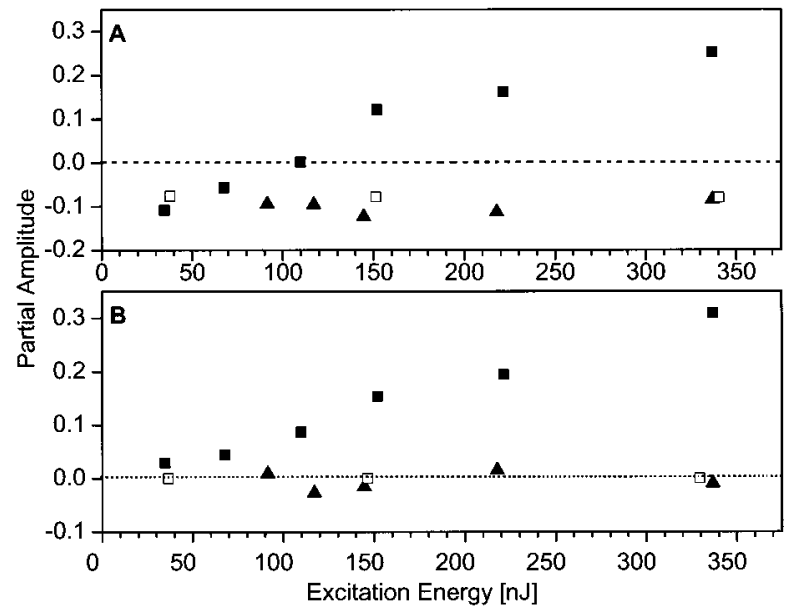

Figure 8. Dependence of the partial amplitude $a_{3}$ of the third component from the laser excitation energy for the compounds G2R4

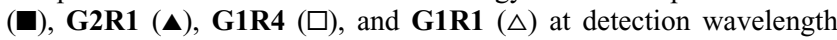
$630 \mathrm{~nm}(\mathrm{~A})$ and $590 \mathrm{~nm}(\mathrm{~B})$.

amplitude offset of the multichromophoric compounds with respect to the monochromophoric compounds is more pronounced for the first-generation compound G1R4 than for the second-generation compound G2R4. This effect can also be observed in Figure 7 in which the amplitude $a_{2}$ is displayed as a function of the excitation energy at the detection wavelengths $630 \mathrm{~nm}$ (Figure 7A) and $590 \mathrm{~nm}$ (Figure 7B). At both detection wavelengths, the curve for the first-generation compound G1R4 has a larger partial positive amplitude than the second-generation compound G2R4.

The partial amplitude $a_{3}$ as a function of the detection wavelength is shown in Figure 5B. The shapes of all of the amplitude spectra of G1R1, G2R1, and G1R4 are almost identical. G2R4 shows the same general shape however at a much larger partial amplitude.

The partial amplitude as a function of the excitation energy is depicted in Figure 8 at the wavelengths $630 \mathrm{~nm}$ (Figure 8A) and $590 \mathrm{~nm}$ (Figure 8B). The partial amplitude of the $40 \mathrm{ps}$ component of G2R4 is clearly dependent on the excitation energy, as opposed to the partial amplitudes, $a_{3}$, of the monochromophoric G1R1 and G2R1, which show no dependence on the excitation energy.
Because of the dependence of $a_{2}$ and $a_{3}$ on the excitation energy in G2R4, these 5 and 40 ps components can be attributed to annihilation processes. The appearance of two annihilation processes in G2R4 relates to the mixture of constitutional isomers, which gives a broader distribution of distances between neighboring chromophores compared to that of G1R4. As a result of this, besides a short (ca. 5 ps) annihilation process occurring between chromophores at short distances, similar to G1R4 but less important in G2R4, an additional annihilation process (ca. 50 ps) is resolved, which can be attributed to interactions between chromophores at longer distances. Two possible structures for isomers with a short- and a long-distance pair of chromophores are depicted in Figure $2 \mathrm{a}, \mathrm{a}^{\prime}$ and $2 \mathrm{~b}, \mathrm{~b}^{\prime}$, respectively. The relative contribution of the short annihilation process indicates approximately $10-15 \%$ of isomers in which the two chromophores are at shorter distances.

The 50 ps component of G2R1 is independent of the laser energy. Probably, it is an analogue to the previously observed component for G1R1, which was found to be due to an intermolecular process. In G2R4, this process might be present but is obscured by the much more important annihilation process. In single-photon-timing experiments, this component is not observed because of the much lower concentration and the much lower power compared to the up-conversion experiments.

\section{Conclusions}

The dynamics of intramolecular energy hopping and singletsinglet annihilation in a series of second-generation polyphenylene dendrimers are quantitatively studied and compared with the corresponding first-generation dendrimers. The energytransfer process has been explained in terms of Förster-type resonance energy transfer. Considering the importance of the dipole-dipole orientation factor, the average $\kappa^{2}$ values have been determined as 2.7 and 1.5 for the G2R4 isomers with a longand short-distance pair of chromophores, respectively. This difference in $\kappa^{2}$ values influences the value of $R_{0}$, and hence, the derived $k_{\text {hopp }}$ has to be considered as an average hopping rate among the chromophores.

In fluorescence up-conversion, the kinetic processes observed in the first and second generation have similar characteristics. Moreover, in the multichromophoric second-generation compound G2R4, a dual annihilation process was observed. The fast annihilation process occurs between a short-distance pair of chromophores comparable in distance to the one in G1R4, while the longer time annihilation process occurs among the more prevalent pair of chromophores at longer distance. The origin of this can be traced back to the distribution of constitutional isomers as a result of the synthesis.

Acknowledgment. M. Lor thanks the "Vlaams instituut voor de bevordering van het wetenschappelijk en technologisch onderzoek" (IWT). The authors gratefully acknowledge the FWO, the Flemish Ministry of Education through Grant GOA 1/96, the EC through the TMR Sisitomas, the Bundesministerium for Education and Research of the Federal Republic of Germany, the Volkswagenstiftung, and the support of DWTC (Belgium) through Grant IUAP-IV-11.

\section{Note Added after ASAP Posting}

This article was released ASAP on 10/10/2001 with minor errors in the text, Figure 4 caption, and equation 6 . The correct version was posted on 11/15/2001. 


\section{References and Notes}

(1) Toppet, S.; Gopidas, K. R.; Leheny, A. R.; Caminati, G.; Turro, N. J.; Tomalia, D. A. J. Am. Chem. Soc. 1991, 113, 7335.

(2) Duan, R.; Miller, L.; Tomalia, D. A. J. Am. Chem. Soc. 1995, 117, 10783.

(3) Hawker, C. J.; Fréchet, J. M. In Step-growth polymers for high performance materials: new synthetic methods; Hendrick, J. L., Lanzani,

G., Eds.; American Chemical Society, Washington, DC, 1996; p 132

(4) Aoi, K. A.; Itah, K.; Okada, M. Macromolecules 1995, 28, 5391

(5) Archut, A.; Vögtle, F. Chem. Soc. Rev. 1998, 27, 233.

(6) Zimmerman, S. C.; Zeng, F. W.; Reichert, D. E. C.; Kolotuchin,

S. V. Science 1996, 271, 1095.

(7) Fréchet, J. M. Science 1994, 263, 1710.

(8) Tomalia, D. A. Top. Curr. Chem. 1993, 165, 193

(9) Morgenroth, F.; Kubel, C.; Müllen, K. J. Mater. Chem. 1997, 7, 1207.

(10) Bosman, A. W.; Janssen, H. M.; Meijer, E. W. Chem. Rev. 1999 99, 1665.

(11) Jansen, J. F. G. A.; De Brabander-Vanden Berg, E. M. M.; Meijer,

E. W. Science 1994, 266, 1226.

(12) Matthews, O. A.; Shipway, A. N.; Stoddart, J. F. Prog. Polym. Sci. 1998, 23, 1 .

(13) Atwood, J. L.; Davies, J. E. C.; Macnicol, D. D.; Vögtle, F.; Lehn, J. M. Comprehensive Supramolecular Chemistry; Pergamon Press: Oxford, U.K., 1996.

(14) Stinson, S. C. Chem. Eng. News 1997, 75, 28

(15) Roberts, J. C.; Bhalgat, M. K.; Zera, T. R. J. Biomed. Mater. Res. 1996, 30, 53.

(16) Twyman, L. J.; Beezer, A. E.; Esfand, R.; Hardy, M. J.; Mitchell, J. C. Tetrahedron Lett. 1999, 40, 1743.

(17) Yeow, E. K. L.; Ghiggino, K. P.; Reek, J. N. H.; Crossley, M. J.; Bosman, A. W.; Schenning, A. P. H.; Meijer, E. W. J. Phys. Chem. B 2000 104, 2596

(18) Adronov, A.; Fréchet, J. M. J. Chem. Commun. 2000, 1710.

(19) Bar-Haim, A.; Klafter, J. J. Lumin. 1998, 76\&77, 197.

(20) Vosch, T.; Hofkens, J.; Cotlet, M.; Köhn, F.; Fujiwara, H.; Gronheid, R.; Van Der Biest, K.; Weil, T.; Herrmann, A.; Müllen, K. Mukamel, S.; Van Der Auweraer, M.; De Schryver, F. C., submitted for publication.

(21) De Belder, G.; Jordens, S.; Lor, M.; Schweitzer, G.; De, R.; Weil, T.; Herrmann, A.; Wiesler, U.-M.; Müllen, K.; De Schryver, F. C. J. Photochem., in press.

(22) De Belder, G.; Schweitzer, G.; Jordens, S.; Lor, M.; Mitra, S.; Hofkens, J.; De Feyter, S.; Van Der Auweraer, M.; Herrmann, A.; Weil, T.; Müllen, K.; De Schryver, F. C. Chem. Phys. Chem. 2001, 1, 49.

(23) Maus, M.; Mitra, S.; Lor, M.; Hofkens, J.; Weil, T.; Herrmann, A.; Müllen, K.; De Schryver, F. C. J. Phys. Chem. A 2001, 105, 3961.
(24) Maus, M.; De, R.; Lor, M.; Weil, T.; Mitra, S.; Wiesler, U.-M.; Herrmann, A.; Hofkens, J.; Vosch, T.; Müllen, K.; De Schryver, F. C. J. Am. Chem. Soc. 2001, 123, 7668

(25) Weil, T.; Wiesler, U.-M.; Herrmann, A.; Bauer, R.; Hofkens, J.; De Schryver, F. C.; Müllen, K. J. Am. Chem. Soc. 2001, 123, 8101.

(26) Spartan Program; Wave Function, Inc.: Irvine, CA, 1999.

(27) Maus, M.; Rousseau, E.; Cotlet, M.; Schweitzer, G.; Hofkens, J.; Van der Auweraer, M.; De Schryver, F. C.; Krueger, A. Rev. Sci. Instrum, 2000, 72,36 .

(28) Program developed in a cooperation between The Management of Technology Institute (Belarusian State University) and The Division of Photochemistry and Spectroscopy (University of Leuven).

(29) O'Connor, D. V.; Phillips, D. Time-Correlated Single Photon Counting; Academic Press: London, 1984; p 252.

(30) Schweitzer, G.; Xu, L.; Craig, B.; De Schryver, F. C. Opt. Commun 1997, 142, 283

(31) Karni, Y.; Jordens, S.; De Belder, G.; Schweitzer, G.; Hofkens, J.; Gensch, T.; Maus, M.; De Schryver, F. C.; Herrmann, A.; Müllen, K. Chem. Phys. Lett. 1999, 310, 73.

(32) Baskin, J. S.; Banares, L.; Pedersen, S.; Zewail, A. H. J. Phys Chem. 1996, 100, 11920.

(33) Nesbitt, D. J.; Field, R. W. J. Phys. Chem. 1996, 100, 12735.

(34) Stratt, R. M.; Maroncelli, M. J. Phys. Chem. 1996, 100, 12981.

(35) Gustavsson, T.; Baldacchino, G.; Mialocq, J. C.; Reekmans, S. Chem. Phys. Lett. 1995, 236, 587.

(36) Jarzeba, W.; Walker, G. C.; Johnson, A. E.; Kahlow, M. A.; Barbara, P. F. J. Phys. Chem. 1988, 92, 7039.

(37) Kimura, Y.; Alfano, J. C.; Walhout, P. K.; Barbara, P. F. J. Phys. Chem. 1994, 98, 3450.

(38) Reynolds, L.; Gardecki, J. A.; Frankland, S. J. V.; Horng, M. L.; Maroncelli, M. J. Phys. Chem. 1996, 100, 10337.

(39) Changenet, P.; Plaza, P.; Martin, M. M.; Meyer, Y. H. J. Phys. Chem. A 1997, 101, 8186.

(40) Changenet, P.; Zhang, H.; Van Der Meer, M. J.; Hellingwerf, K J.; Glasbeek, M. Chem. Phys. Lett. 1998, 282, 276.

(41) Harcourt, R. D.; Ghiggino, K. P.; Scholes, G. D.; Steer, P. D. J. Chem. Phys. 1998, 109, 1310.

(42) Paillotin, G.; Swenberg, C. E.; Breton, J.; Geacintov, N. E. Biophys. J. 1979, 25, 513

(43) Valkunas, J.; Gulbinas, V. J. Photochem. Photobiol. 1997, 66, 628.

(44) Westerhuis, W. H. J.; Vos, M.; Van Grondelle, R.; Amesz, J.; Niederman, R. A. Biochim. Biophys. Acta 1998, 1366, 317.

(45) Ruseckas, A.; Theander, M.; Valkunas, L.; Andersson, M. R. Inganas, O.; Sundstrom, V. J. Lumin. 1998, 76, 474.

(46) Scheblykin, G.; Varnavsky, O. P.; Bataiev, M. M.; Sliusarenko, O.; Van Der Auweraer, M.; Vitukhnovsky, A. G. Chem. Phys. Lett. 1998, $298,341$. 